\title{
EFFECTS OF TREADMILL TRAINING ON MICROVASCULAR REMODELING IN THE RAT FOLLOWING SPINAL CORD INJURY
}

Authors: Roger W.P. Kissane, PhD, ${ }^{1}$ Oliver Wright, MBChB,${ }^{1}$ Yazi D. Al'Joboori, PhD, ${ }^{1}$ Paulina Marczak, BSc, ${ }^{1}$ Ronaldo M. Ichiyama, PhD, ${ }^{1}$ and Stuart Egginton, PhD, ${ }^{1}$

Author Affiliation: ${ }^{1}$ School of Biomedical Sciences, University of Leeds, Leeds, LS2 9JT, UK.

The authors are grateful to the School of Biomedical Sciences, University of Leeds for provision of a scholarship to R.W.P.K. This study was also supported by a grant from the International Spinal Research trust (Project No. NRB107) for Y.D.A and RMI, and the Medical Research Council (MR/K022911/1) for RMI.

Abstract word count: 150

Manuscript word count: 4092

Corresponding Author: Stuart Egginton. Email: s.egginton@leeds.ac.uk

Running Title: Microvascular remodeling in SCI

Ethical Statement: We confirm that we have read the Journal's position on issues involved in ethical publication and affirm that this report is consistent with those guidelines.

Conflict of Interest: None of the authors has any conflict of interest to disclose 


\section{Effects of treadmill training on microvascular remodeling in the rat following spinal cord injury}

\section{ABSTRACT}

Introduction: The morphological characteristics of skeletal muscles innervated caudal to a spinal cord injury (SCl) undergo dramatic phenotypic and microvascular changes.

Method: Female Sprague Dawley rats received a severe contusion at thoracic level 9/10, and randomly assigned to locomotor training (TR), epidural stimulation (ES) or a combination of the two (CB) treatment groups. Fibre type composition and capillary distribution were assessed in phenotypically distinct compartments of the tibialis anterior.

Results: SCI induced a shift in Type II fibre phenotype from oxidative to glycolytic $(P<0.05)$ as well as capillary loss within the oxidative core and glycolytic cortex; the CB treatment best maintained capillary supply within both compartments.

Discussion: The angiogenic response of CB training improved capillary distribution across the muscle, becoming spatially more homogeneous and decreasing mean capillary supply area, potentially improving oxygenation. There is an important role for weight bearing training in maintaining the oxidative phenotype of muscle following $\mathrm{SCl}$.

Key Words: Spinal Cord Injury, Skeletal Muscle, Epidural Stimulation, Treadmill Training, Capillary Domain Area, Angiogenesis. 


\section{INTRODUCTION}

Spinal cord injury (SCI) is a traumatic life changing injury, associated with neurological impairment and paralysis of skeletal muscles that are innervated caudal to the lesion site. Following some $\mathrm{SCl}$ there is a dramatic atrophy of skeletal muscle in both animals ${ }^{1-3}$ and humans ${ }^{4-6}$. This reduction in muscle mass is accompanied by a shift from an oxidative to a highly glycolytic, fatigable phenotype ${ }^{7-9}$. In addition, over a chronic time period post injury fibre size has been shown to first stabilise and then return towards baseline ${ }^{10}$. Largely unreported, the microvascular supply also undergoes remodelling, typically in the form of capillary rarefaction. This reduces the diffusive capacity to deliver oxygen and fuel to working muscles, which is correlated with impaired fatigue resistance ${ }^{10,11}$. However, the lack of correlation between blood flow and muscle fatigue in SCI implicates microvascular rather than macrovascular limitations as a target for therapeutic strategies ${ }^{12}$. A combination of increased glycolytic phenotype, increased fibre size and reduced capillary density contributes to the reduced functional capacity to sustain activity. These morphometric and functional outcomes are consistent across a variety of animal models and SCI in humans.

Therapeutic interventions for the recovery of locomotor function often overlook the importance of skeletal muscle. Locomotor training has been shown to ameliorate the loss in muscle mass and function in spinal cord injured humans ${ }^{11,13}$ and animals ${ }^{14-17}$. Treadmill locomotor training has been shown to improve microvascular supply in denervated skeletal muscle of animals ${ }^{18}$ a potential translational effect relevant to that of spinal cord injured muscle. Alternative therapeutic techniques include direct stimulation of the spinal cord surface, i.e. epidural stimulation (ES), which has been shown to elicit hind limb stepping in a rhythmic, locomotor fashion, in both animals ${ }^{19-21}$ and humans ${ }^{22}$. An advantage of ES compared with direct or indirect muscle stimulation is that it allows a more natural, coordinated recruitment of synergistic muscle groups ${ }^{23,20}$, thus inducing less muscle fatigue ${ }^{24}$. Although ES can effectively improve locomotor function, its ability to modulate skeletal muscle phenotype and 
microvascular supply has not been determined. These strategies as standalone treatments have their own distinct adaptive benefits, and therefore present a strong rationale for their use in a combination therapy to utilise the different adaptive mechanisms.

In this pilot study, we determined the phenotypic transformation of skeletal muscle in response to epidural stimulation and treadmill locomotor training, as stand-alone treatments and in combination. In addition, we quantified muscle capillary content, as it is often overlooked that the microvascular supply is integral for normal muscle function. It is unknown what effect the changes in fibre area and rarefaction of the capillary bed will have on the supply capacity of the muscle. As commonly used measures are scale-dependent, we additionally used local capillary analysis to incorporate fibre area and quantify the supply area of individual capillaries. Thus, spatial distribution of capillaries reflects the close packing of fibres with differing girth, and the resultant heterogeneity of intercapillary diffusion distances markedly affects peripheral oxygen transport ${ }^{25-27}$. In rodents, distinct fibre types also differ in size, such that estimates of capillary supply to individual fibres needs to accommodate the complex interaction of such influences; this is not possible with more traditional indices ${ }^{28,29}$. In general, muscle inactivity tends to increase the area of tissue supplied by individual capillaries, and reduce capillary supply to individual fibres, both of which will reduce exercise tolerance ${ }^{25,30}$.

We hypothesised that the application of a single training stimulus would reduce the degree of fibre atrophy and alleviate the decrease in global and local capillary supply. We reasoned the combination of both treatments would have an additive response, and hence improve capacity for routine physical activity that is fundamental to quality of life. 


\section{METHODS}

\section{Ethical approval}

Twenty female adult Sprague Dawley rats were used, weighting 210-240g (obtained from Charles River Laboratories). Animals were individually housed on a 12 hour light/dark cycle with ad libitum access to food and water. All animal procedures were approved by the UK Home Office and University of Leeds Animal Welfare and Ethics Committee, in accordance with UK Animals (Scientific Procedures) Act 1986.

\section{Overview of experimental timeline}

Briefly, animals received a severe contusion injury and implantation of epidural electrodes at day zero. Animals were then randomly assigned to experimental treatment groups; cage control (CG), locomotor training only (TR), epidural stimulation only (ES) or a combination of both TR and ES (CB). Training began seven days post injury and continued for eight weeks. Twenty minutes of training was delivered five days a week, using a distributed training format; ten minutes of training, ten minutes of rest, ten minutes of training. Animals were sacrificed three weeks following the end of training. Intact weight matched controls (CT) were used to compare the effect of $\mathrm{SCl}$ on baseline physiological factors.

\section{Spinal cord injury}

Animals were anaesthetised with Isoflurane: 5\% IsoFlo® (Zoetis, UK Ltd, London, UK) at 2 L. $\min ^{-1} \mathrm{O}_{2}$ flow induction, and maintained with $2.5 \%$ throughout surgery. An incision was made from $\sim$ T4 to $\sim \mathrm{S} 5$ and cleared of fascia and muscle around the laminectomy site. Partial laminectomies were performed at vertebral levels of the contusion (T10), and epidural implant sites (T12/13 and L2). A severe midline contusion injury was made at T9/10 ${ }^{31}$ using the Infinite Horizon impactor at 250 kdyn (Precision Systems \& Instrumentation, Lexington, KY, USA). Immediately following the injury, chondroitinase $A B C$ was injected into the spinal cord $1 \mathrm{~mm}$ rostral and caudal to the lesion through a lentiviral vector. The chondroitinase $A B C$ gene was 
produce as previously described by Bartus et al. ${ }^{32}$ and subcloned into a lentiviral vector by Penn Vector Core (University of Pennsylvania). The promotor was mouse phosphoglycerate kinase 1 (mPGK1) ${ }^{32,33}$ and the viral titre was $1.51 \times 10^{10}$ genome copies per millilitre $(\mathrm{GC} / \mathrm{ml})$ determined by real-time PCR. This was delivered to all groups to maximize plasticity and the effects of each treatment ${ }^{34-36,33}$.

\section{Epidural stimulation}

Stimulation was delivered via stainless steel wires (AS632, Cooner Wire, Chatsworth, CA, USA) implanted on the midline of the spinal cord facing the dorsal aspect over segments L2 and $\mathrm{S} 1{ }^{23}$. The wires were secured with sutures (Ethilon 9.0, Ethicon, Johnson \& Johnson Medical Ltd, New Brunswick, NJ, USA) either side of the notch to the spinal dura. The implanted wires were subcutaneously tunneled to a skull mounted head plug (MCS-16SS; Omnetics, Minneapolis, MN, USA), and stimulated via a stimulation (S88X stimulator; AstroMed®, Inc. Grass instruments, Middleton, WA, USA) and isolation unit (SIU-V Isolation unite; Astro-Med®, Inc. Grass instruments, Middleton, WA, USA). Animals were suspended in a custom-made jacket during training. Stimulation was delivered using continuous rectangular pulses (200 $\mu \mathrm{s}$ in duration at $40 \mathrm{~Hz}$, with no delay) ${ }^{20,23,37}$ with a voltage sufficient to activate the hind limb muscles.

\section{Locomotor training}

All animals undergoing locomotor training on a small animal treadmill were secured by a custom-made jacket to provide body weight support, and allow for manual manipulation of hind limbs during stepping. Body weight support was optimized for each session to facilitate stepping, judged by unhampered swing phase, and support the trunk during progression from bipedal to quadrupedal stepping (Rodent Robot 3000, Robomedica, Inc., Irvine, USA). Animals were initially trained at $7 \mathrm{~cm} \mathrm{~s}^{-1}$, increasing in speed to $21 \mathrm{~cm} \mathrm{~s}^{-1}$ at week four, and maintained so for the remaining four weeks. 


\section{Tissue preparation}

Spinal cord injured animals were sacrificed using a terminal dose of pentobarbitone sodium 20\% (Pentoject, Animalcare Ltd, York, UK), while intact animals were culled through cerebral concussion and cervical dislocation. Tibialis anterior (TA) muscles were dissected carefully and a mid-belly steak snap frozen in liquid nitrogen cooled isopentane. Serial cryostat sections $(10 \mu \mathrm{m})$ were cut at $-20^{\circ} \mathrm{C}$ and attached to poly lysine coated slides (VWR International), and stored at $-20^{\circ} \mathrm{C}$ until staining.

\section{Immunohistochemistry}

Muscle fibre types were distinguished using two monoclonal anti-MHC antibodies (Developmental Studies Hybridoma Bank, University of lowa): BA-D5 for Type I fibres labelled with Alexa Fluor 555 Goat Anti-Mouse IgG (Life Technology, A21422) and SC-71 for Type Ila (fast oxidative, glycolytic) labelled with Alexa Fluor 488 Rabbit Anti-Mouse IgG (Life Technology, A11059). The remaining unstained fibres are considered to be Type Ilb (fast glycolytic) ${ }^{38-40}$. Muscle fibre boundaries were labelled with an anti-laminin antibody (Sigma, L9393), which was biotinylated against with Anti-Rabbit IgG (Vector Labs, BA1000) and labelled with Streptavidin, Pacific Blue Conjugate (Life Technology, S11222). Capillaries were simultaneously identified using Griffonia simplicifolia lectin-1 (Vector Laboratories, UK).

\section{Morphometric analysis}

Two metabolically distinct regions within the TA were studied, the medial oxidative core and the lateral glycolytic cortex, utilising five fields of interest each $0.145 \mathrm{~mm}^{2}$ (Supplementary Fig. 1). Global morphometric indices provide an overview of mean tissue composition; for capillary supply this includes the numerical capillary to fibre ratio $(C: F)$ and capillary density $\left(C D, m^{-}\right.$ $\left.{ }^{2}\right)$, and for fibre type composition involved fibre cross sectional area (CSA, $\left.\mu \mathrm{m}^{2}\right)$ numerical $\left(N_{N}\right)$ and areal density $\left(A_{A}\right)$. Subsequently, determining the local geometric tissue supply regions for individual capillaries (capillary domain area, $\mu \mathrm{m}^{2}$ ) allowed fibre-specific supply area to be calculated ${ }^{25}$; The local capillary to fibre ratio (LCFR, or 'capillary equivalent' of supply) is 
calculated from the cumulative intersection of domains with individual fibres, whereas the local capillary density (LCD) represents LCFR normalised for fibre CSA and hence represents a scale-independent supply index. Spatial heterogeneity of capillary location follows the distribution of domain area; as this is a log-normal function an appropriate index of variance is provided by LogSD.

\section{Statistical analysis}

All data are expressed as mean \pm S.E.M. (n). A one-way analysis of variance (ANOVA) was used to determine differences between groups, using Tukey post-hoc testing for individual comparison when there were significant differences, set at $P<0.05$. Least-squares regression slopes showing the relationships between; LCFR vs. CSA and LCD vs. CSA, were determined for pooled data, and the significance of these slopes were calculate as described by ${ }^{41}$. Animal numbers (n) in the groups were as follows: CT (4), CG (4), TR (5), ES (3) and CB (4), see Supplementary Table 1 for more details. 


\section{RESULTS}

\section{Prevention of capillary rarefaction in spinal cord injury}

Twelve weeks of SCI resulted in a significant decrease in C:F in both the core $(1.54 \pm 0.04$ vs. $2.14 \pm 0.07, P<0.001)$ and cortex $(1.27 \pm 0.06$ vs. $1.57 \pm 0.02, P=0.001)$, for CG vs. CT (Fig. $2 A, B) . C D$ was also significantly decreased in the two regions of the $T A$, with no significant difference in CSA (Fig. 1). TR had a significant decrease in C:F in the core $(1.74 \pm 0.10$, $\mathrm{P}=0.047)$ and moderate decrease in the cortex $(1.38 \pm 0.03, \mathrm{P}=0.082)$ compared to $\mathrm{CT}$. Similarly ES tended to be lower in the core $(1.71 \pm 0.08, P=0.052)$ and cortex $(1.38 \pm 0.02$, $\mathrm{P}=0.184)$ compared to $\mathrm{CT}$. CD was significantly decreased in both the core and cortex following TR and ES (Fig. 1C, D). CB treatment showed a significant maintenance of capillarity, with a C:F close to that of $C T$, significantly higher in the core $(2.03 \pm 0.04, P=0.002)$ and cortex $(1.50 \pm 0.06, P=0.01)$ vs. $C G$. The preservation in $C: F$ was not mirrored in $C D$, while CSA showed a significant increase in only the cortex of CB treated animals CB vs. CT (Fig. 1E, F).

\section{Fibre type composition following SCI}

Type I fibres differed the most among groups with ES treated animals having significantly larger average fibre areas than CT, CG and CB animals (Fig. 2A). Numerical fibre type composition in the CG core showed a significant decrease in Type lla fibres $(0.20 \pm 0.02$ vs. $0.40 \pm 0.03, P=0.002)$ and increase in Type $\mathrm{Ilb}(0.75 \pm 0.03$ vs. $0.57 \pm 0.04, P=0.038)$ compared to CT (Fig. 2B). This shift in numerical composition was mirrored in relative fibre area, while there were no significant changes in mean fibre size. TR and ES both showed significant decreases in core numerical Type Ila fibre composition, with slight increases in Type I and IIb when compared with intact animals. CB therapy displayed similar significant decreases in Type lla numerical composition compared to CT, however there was a significant increase in numerical and areal density for Type I compared to CT and CG (Fig. 2B, C). 


\section{Heterogeneity of capillary supply area}

The frequency distribution displays a large right shift in median capillary domain area for CG animals compared with CT, 1,146 vs. $712 \mu \mathrm{m}^{2}$ and 2,111 vs. $1,203 \mu \mathrm{m}^{2}$, for the core and cortex, respectively (Fig. 3A, B). The shift in distribution corresponds with a significant reduction in the LogSD for the core $(0.13 \pm 0.004$ vs. $0.15 \pm 0.01, P=0.034)$ and cortex $(0.13$ \pm 0.01 vs. $0.20 \pm 0.01, P<0.001$ ) (Fig. $3 C, D$ ). Neither individual treatment (TR or ES) altered the capillary domain distribution in relation to $C G$, with equivalent median capillary domain areas across both core and cortex (Fig. 3A, B). Capillary heterogeneity in the TR group significantly increased in the core relative to $C G(0.16 \pm 0.004$ vs. $0.13 \pm 0.004, P=0.001$; Fig. 3C, D), while in the cortex both TR and ES were not significantly different from CG. The CB group demonstrated a leftward shift towards CT, with an intermediate median capillary domain area situated between CT and CG (Fig. 3A, B). The heterogeneity of the CB group was significantly reduced in the core when compared to CT, TR and ES (Fig. 3C, D). Distribution of fibre size are presented in Figure 3E-F and heterogeneity of fibre size (based on LogSD of fibre CSA) across TA compartments in Figure 3G-H. Following SCI fibre size becomes more homogeneous compared to intact controls in the core $(0.14 \pm 0.002$ vs. $0.16 \pm 0.008, P=0.474)$ and cortex $(0.14 \pm 0.005$ vs. $0.18 \pm 0.005, P=0.052)$. ES significantly decreased CSA heterogeneity when compared to CT and TR animals in the core (Fig. 3G), while ES, TR and CB treatments showed a shift to a more homogeneous CSA distribution in the cortex (Fig. $3 \mathrm{H})$.

\section{Local capillary supply}

LCFR and LCD for the major fibre type compositions in the core and cortex of the TA are presented (Table 1), and the correlation with fibre size are shown in Figure 4. Local capillary supply was significantly reduced in CG compared to CT, with lower LCFR and LCD across the three fibre types within the core (all, $\mathrm{P}<0.02$ ), and in the cortex Type Ilb showing decreased LCFR $(P=0.344)$ and LCD $(P<0.001)$. Following TR, the LCFR and LCD remained significantly 
decreased for the three major fibre types in the core, as well as the Type Ilb fibres in the cortex, when compared to CT with no significant improvement relative to CG. The ES training attenuated some of the reductions in LCFR across Type I and Ila fibres, while LCD remained significantly reduced across the core and cortex, compared to CT. The CB treatment group showed re-establishment of local capillary supply compared to CG, with similar LCFR values for both Type II fibres in the core and Type Ilb fibres within the cortex to that of CT.

The correlative data for local capillary supply (LCFR and LCD) relative to CSA showed significant shifts across all fibres types following $\mathrm{SCl}$ (all, $\mathrm{P}<0.05$ ). ES alone was unable to return the scaling of LCFR and LCD to levels seen in intact animal, while TR further disrupted the typical relationship between capillary supply area and CSA (Fig. 4, Table 2). The CB treatment rectified the relationship across Type lla fibres, but while Type Ilb LCFR was normalised ( $P=$ n.s., vs. CT and vs. CGCT), LCD was not $(P<0.05$, vs. CT and vs. CGCT). 


\section{DISCUSSION}

There are clear differences in muscle adaptations with standalone and combinatorial treatments following SCI. Epidural stimulation does not rectify the glycolytic shift seen with $\mathrm{SCl}$, whereas locomotor training appears to alleviate it. Neither ES nor TR were sufficient to evoke an angiogenic response, and fibre hypertrophy further reduced CD across the muscle. CB treatment maintained microvascular supply near to that of intact animals, across almost all measures of capillarity, while TR or ES alone did not. Locomotor training appears to have the greatest effect on CSA, which resulted in a further reduction in LCD. Overall, CB treatment best preserved microvascular supply, maintaining capillary distribution near to that of intact levels, and best attenuated the SCl-induced slow-to-fast shift in fibre type composition, compared to individual treatments alone.

\section{Global morphometrics}

Regional comparisons within the TA muscle quantified the extent of capillary rarefaction following contusion injury; this reduced number of capillaries combined with a well maintained CSA resulted in a $35 \%$ decrease in CD across the muscle, and suggests an impaired functional aerobic capacity of the TA. Standalone treatments of ES or locomotor training showed no observable changes in capillary content, while the hypertrophic response within the core and cortex further reduced CD. However, the combination of these training modalities was sufficient to maintain capillary supply $(\mathrm{C}: \mathrm{F})$ within both metabolic compartments of the TA, although a global hypertrophic response reduced the functionally relevant CD. The combination of treatments appears to ameliorate capillary rarefaction that result from $\mathrm{SCl}$, but understanding the functional relevance of an angiogenic response cannot be inferred from the CD alone ${ }^{30}$.

Muscles typically begin to atrophy a few days after $\mathrm{SCl}$, which varies in extent depending on initial muscle phenotype and injury type ${ }^{10}$. The time course and degree of atrophy within $\mathrm{SCl}$ 
models differs greatly, with complete transections generating the greatest level of atrophy ${ }^{10}$. Oxidative fibres typically atrophy prior to glycolytic muscle fibres, and normalise at different times $^{1,3}$, with some returning to control levels after 6-8 weeks ${ }^{1}$. This highlights a benefit of using the TA muscle for histological analysis, as we are able to characterise metabolically distinct regions and their response within one functionally integrated muscle.

Following SCI the average CSA in our study did not differ from weight matched intact animals. There was a clear shift in core phenotype to an increasingly glycolytic form, through a reduction in Type Ila fibres, and increased Type Ilb. All three treatment groups displayed a global hypertrophic response within both compartments of the TA. ES showed a transformation of Type Ila fibres to Type Ilb, with Type I numerical composition being maintained. This decrease in number dramatically reduced the overall oxidative proportion of the TA core. Conversely, locomotor training and the combination therapy better maintained oxidative composition, with higher numerical and areal densities of Type I and Ila fibres. This suggests an integral role for weight bearing training and afferent stimulation of normal locomotion in order to maintain oxidative phenotype.

Initially, our data suggest that microvascular rarefaction may precedes changes in muscle fibre area, however, the chronic nature of this work means it is unlikely. It is likely that at the acute stage of injury skeletal muscle atrophy precedes some microvascular change, as a decreased fibre area would result in an increased CD ${ }^{42}$. The skeletal muscle in turn would become excessively capable to supply oxygen and fuel, and drive the pruning of capillaries. Finally, the normalisation of CSA would further reduce microvascular density.

\section{Local capillary supply}

Heterogeneity in capillary spacing differs greatly between the two compartments of the TA, with the core having a lower average capillary domain area and narrower distribution ${ }^{30}$, 
conditions known to optimise oxygen diffusion ${ }^{26}$. The summed proportion of capillary domains normalised to fibre area (LCD) demonstrate that local capillary supply is higher in oxidative fibres than glycolytic ${ }^{28}$, irrespective of fibre size ${ }^{28,29,27}$, in intact control animals.

Capillary rarefaction induced by $\mathrm{SCl}$ decreases local capillary supply, and appears to predominantly target oxidative fibres (35\% decrease in LCFR of Type I and Ila fibres, while Type Ilb decreased 30\%), generating an overall lower LCD to that seen in intact mixed muscle phenotypes ${ }^{29,27}$. The decreased heterogeneity of the capillary domains is in part linked to the increased homogeneity of the fibre area. Through normalization of fibre area and selective rarefaction of the microvasculature the muscle preserves an homogeneous LCD, irrespective of fibre type, deviating from natural metabolic influence of muscle phenotype.

In contrast, apparent rarefaction of capillaries across the TA of epidural stimulated animals was not accompanied by a significant decrease in capillary supply heterogeneity, despite a more homogeneous fibre size distribution. The global effect of ES across the TA was to further reduce LCD for glycolytic fibres when compared to CG animals. Locomotor training increased capillary domain area across both the core and cortex, and maintained a normal level of heterogeneity of capillary distribution and fibre size within the core, despite a severe rarefaction. Maintaining spatial heterogeneity retained the normal allometric relationship of LCD for oxidative fibres, but the significant hypertrophy of glycolytic fibres further reduced the proportion of oxygen supply by individual capillaries to levels below that of $\mathrm{SCl}$ animals.

The simultaneous application of epidural stimulation and locomotor training proved to be the most effective in inducing structural remodelling that would favor improved oxygen transport kinetics. Through maintenance of an oxidative phenotype and targetted angiogenesis the combination therapy was sufficient to shift the distribution of capillary domains closer towards intact levels, reducing average domain area while simultaneously decreasing heterogeneity 
of supply. The combination strategy increased LCFR across all fibre types, when compared to $\mathrm{SCl}$ animals, preserving the LCFR to near intact levels.

Under normal physiological conditions, capillary spacing heterogeneity is similar across different muscle phenotypes, and across a variety of species. This is likely to be a result of local feedback from individual fibres, to optimize diffusive oxygen supply to meet demand ${ }^{25}$. Where a reduction in heterogeneity is seen, it would suggests a dissociation between signals regulating capillary rarefaction and alteration in fibre composition, which would in turn suggesting no functional benefit in terms of diffusive capacity. Both interventions that contained load bearing elements maintained near normal fibre heterogeneity, which interacts with microvascular remodelling to optimize peripheral $\mathrm{O}_{2}$ transport. A combination of $\mathrm{ES}$ and locomotor training maximises this beneficial outcome.

\section{Limitations}

While adopting local capillary supply indices improving data resolution to maximise output from extensive and costly experimental interventions, it is possible that observed trends (e.g. change in CSA, fibre and capillary heterogeneity) may prove to be statistically significant on further replication. In addition, the nature of the interventions makes it difficult to accurately weight match across groups, but the indices adopted will minimize any scale dependency.

Microvascular remodelling may be indirectly stimulated by muscle regeneration, or interventions may directly improve microvascular supply, and the ambiguity of some indices used to quantify microvascular response to therapeutic interventions is well known ${ }^{30}$. The rationale for adopting less commonly used metrics reported in this paper is that quantifying multiple indices, allowing for varying influences on microvascular supply, provides a better position from which to identify potential drivers of remodelling. 
Finally, this study describes treatment of an animal model of spinal cord injury, and implications for the care of human patients with $\mathrm{SCl}$ may be questioned. However, this approach allows some mechanistic insight, and testing of alternate therapeutic strategies, not feasible with human subjects. For fundamental biological processes the translatability of findings has been demonstrated to be robust; e.g., we have established common principles of angiogenesis using mice, rats, horses, and humans ${ }^{43}$. Our conclusions regarding the benefit of weight bearing training during rehabilitation, in order to ameliorate muscle dysfunction following $\mathrm{SCl}$, should therefore be of interest to the clinical community.

\section{Conclusions}

As exercise tolerance is heavily influenced by enzymatic oxidative capacity and the microvascular delivery of essential substrates. It is therefore important to assess both oxygen demand and supply, in order to gain an integrative perspective on the skeletal muscle response to $\mathrm{SCI}$. While Individual treatments increased the heterogeneity of muscle capillary supply, the dramatic uncoupling between fibre area and local capillary supply suggests a reduced efficiency to deliver oxygen to the periphery, when compared to that of SCI animals. However, combination therapy showed the most promising adaptive remodelling, improving the capacity to deliver oxygen through increasing homogeneity of capillary supply, rather than increasing density of the microvascular network. It may be that during pathological remodelling and rarefaction in $\mathrm{SCl}$ the local capillary supply becomes tightly coupled to oxidative capacity, matching supply to demand, in contrast to the relationship seen in intact muscle ${ }^{44}$. 


\section{Abbreviations}

CB Combination training

CD Capillary density

C:F Capillary to fibre ratio

CG Spinal cord Injured cage controls

CSA Fibre cross-sectional area

CT Intact controls

ES Epidural stimulation

LCD Local capillary density

LCFR Local capillary to fibre ratio

SCI Spinal cord injury

TA Tibialis anterior

TR Locomotor training 


\section{References}

1. Durozard D, Gabrielle C, Baverel G. Metabolism of rat skeletal muscle after spinal cord transection. Muscle \& Nerve 2000;23(10):1561-1568.

2. Hiraizumi Y, Fujimaki E, Tachikawa T. Long-term morphology of spastic or flaccid muscles in spinal cord-transected rabbits. Clinical Orthopaedics and Related Research 1990;260:287-296.

3. Landry E, Frenette J, Guertin PA. Body weight, limb size, and muscular properties of early paraplegic mice. Journal of Neurotrauma 2004;21(8):1008-1016.

4. Lotta S, Scelsi R, Alfonsi E, Saitta A, Nicolotti D, Epifani P, Carraro U. Morphometric and neurophysiological analysis of skeletal muscle in paraplegic patients with traumatic cord lesion. Spinal Cord 1991;29(4):247-252.

5. Castro MJ, Apple Jr DF, Hillegass EA, Dudley GA. Influence of complete spinal cord injury on skeletal muscle cross-sectional area within the first 6 months of injury. European Journal of Applied Physiology and Occupational Physiology 1999;80(4):373-378.

6. Round JM, Barr FM, Moffat B, Jones DA. Fibre areas and histochemical fibre types in the quadriceps muscle of paraplegic subjects. Journal of the Neurological sciences 1993;116(2):207-211.

7. Otis JS, Roy RR, Edgerton VR, Talmadge RJ. Adaptations in metabolic capacity of rat soleus after paralysis. Journal of Applied Physiology 2004;96(2):584-596.

8. Hutchinson KJ, Linderman JK, Basso DM. Skeletal muscle adaptations following spinal cord contusion injury in rat and the relationship to locomotor function: a time course study. Journal of Neurotrauma 2001;18(10):1075-1089.

9. Huey KA, Roy RR, Baldwin KM, Edgerton VR. Temporal effects of inactivty on myosin heavy chain gene expression in rat slow muscle. Muscle \& Nerve $2001 ; 24(4): 517-526$.

10. Biering-Sørensen B, Kristensen IB, Kjaer M, Biering-Sørensen F. Muscle after spinal cord injury. Muscle \& Nerve 2009;40(4):499-519. 
11. Crameri R, Weston A, Climstein M, Davis G, Sutton J. Effects of electrical stimulationinduced leg training on skeletal muscle adaptability in spinal cord injury. Scandinavian Journal of Medicine \& Science in Sports 2002;12(5):316-322.

12. Olive JL, Slade JM, Dudley GA, McCully KK. Blood flow and muscle fatigue in SCI individuals during electrical stimulation. Journal of Applied Physiology 2003;94(2):701-708.

13. Giangregorio LM, Webber CE, Phillips SM, Hicks AL, Craven BC, Bugaresti JM, McCartney N. Can body weight supported treadmill training increase bone mass and reverse muscle atrophy in individuals with chronic incomplete spinal cord injury? Applied Physiology, Nutrition, and Metabolism 2006;31(3):283-291.

14. Dupont-Versteegden EE, Murphy RJ, Houlé JD, Gurley CM, Peterson CA. Mechanisms leading to restoration of muscle size with exercise and transplantation after spinal cord injury. American Journal of Physiology-Cell Physiology 2000;279(6):C1677C1684.

15. Roy RR, Talmadge RJ, Hodgson JA, Oishi Y, Baldwin KM, Edgerton VR. Differential response of fast hindlimb extensor and flexor muscles to exercise in adult spinalized cats. Muscle \& Nerve 1999;22(2):230-241.

16. Roy RR, Talmadge RJ, Hodgson JA, Zhong H, Baldwin KM, Edgerton VR. Training effects on soleus of cats spinal cord transected (T12-13) as adults. Muscle \& Nerve 1998;21(1):63-71.

17. Battistuzzo CR, Rank MM, Flynn JR, Morgan DL, Callister R, Callister RJ, Galea MP. Effects Of treadmill training on hindlimb muscles of spinal cord-injured mice. Muscle \& Nerve 2017;55(2):232-242.

18. Jakubiec-Puka A, Sławińska U, Rędowicz MJ, Biral D, Łapińska I, Chomontowska H, Karczewska E, Krawczyk K, Bilski H, Pliszka B. Influence of locomotor training on the structure and myosin heavy chains of the denervated rat soleus muscle. Neurological Research $2008 ; 30(2): 170-178$. 
19. Gerasimenko YP, Avelev V, Nikitin O, Lavrov I. Initiation of locomotor activity in spinal cats by epidural stimulation of the spinal cord. Neuroscience and Behavioral Physiology 2003;33(3):247-254.

20. Ichiyama R, Gerasimenko YP, Zhong H, Roy R, Edgerton V. Hindlimb stepping movements in complete spinal rats induced by epidural spinal cord stimulation. Neuroscience Letters 2005;383(3):339-344.

21. Iwahara T, Atsuta Y, Garcia-Rill E, Skinner R. Locomotion induced by spinal cord stimulation in the neonate rat in vitro. Somatosensory \& Motor Research 1991;8(3):281-287.

22. Minassian K, Jilge B, Rattay F, Pinter M, Binder H, Gerstenbrand F, Dimitrijevic MR. Stepping-like movements in humans with complete spinal cord injury induced by epidural stimulation of the lumbar cord: electromyographic study of compound muscle action potentials. Spinal Cord 2004;42(7):401-416.

23. Ichiyama RM, Courtine G, Gerasimenko YP, Yang GJ, van den Brand R, Lavrov IA, Zhong H, Roy RR, Edgerton VR. Step training reinforces specific spinal locomotor circuitry in adult spinal rats. The Journal of Neuroscience 2008;28(29):7370-7375.

24. Bamford J, Putman C, Mushahwar V. Intraspinal microstimulation preferentially recruits fatigue-resistant muscle fibres and generates gradual force in rat. The Journal of Physiology 2005;569(3):873-884.

25. Al-Shammari AA, Gaffney EA, Egginton S. Modelling capillary oxygen supply capacity in mixed muscles: Capillary domains revisited. Journal of Theoretical Biology 2014;356:4761.

26. Egginton S, Gaffney E. Experimental Physiology-Review Article: Tissue capillary supply-it's quality not quantity that counts! Experimental Physiology 2010;95(10):971-979.

27. Degens H, Turek Z, Hoofd L, Van't Hof M, Binkhorst R. The relationship between capillarisation and fibre types during compensatory hypertrophy of the plantaris muscle in the rat. Journal of Anatomy 1992;180(Pt 3):455. 
28. Gray SD, Renkin EM. Microvascular supply in relation to fiber metabolic type in mixed skeletal muscles of rabbits. Microvascular research 1978;16(3):406-425.

29. Egginton S, Ross H. Influence of muscle phenotype on local capillary supply. Oxygen Transport to Tissue XI: Springer; 1989. p 281-291.

30. Egginton S. Morphometric analysis of tissue capillary supply. Vertebrate Gas Exchange: Springer; 1990. p 73-141.

31. Scheff SW, Rabchevsky AG, Fugaccia I, Main JA, Lumpp Jr JE. Experimental modeling of spinal cord injury: characterization of a force-defined injury device. Journal of Neurotrauma 2003;20(2):179-193.

32. Bartus K, James ND, Didangelos A, Bosch KD, Verhaagen J, Yáñez-Muñoz RJ, Rogers JH, Schneider BL, Muir EM, Bradbury EJ. Large-Scale Chondroitin Sulfate Proteoglycan Digestion with Chondroitinase Gene Therapy Leads to Reduced Pathology and Modulates Macrophage Phenotype following Spinal Cord Contusion Injury. The Journal of Neuroscience 2014;34(14):4822-4836.

33. Zhao R-R, Muir EM, Alves JN, Rickman H, Allan AY, Kwok JC, Roet KCD, Verhaagen J, Schneider BL, Bensadoun J-C, Ahmed SG, Yáñez-Muñoz RJ, Keynes RJ, Fawcett JW, Rogers JH. Lentiviral vectors express chondroitinase ABC in cortical projections and promote sprouting of injured corticospinal axons. Journal of Neuroscience Methods 2011;201(1):228238.

34. Bradbury EJ, Moon LDF, Popat RJ, King VR, Bennett GS, Patel PN, Fawcett JW, McMahon SB. Chondroitinase ABC promotes functional recovery after spinal cord injury. Nature 2002;416:636.

35. García-Alías G, Barkhuysen S, Buckle M, Fawcett JW. Chondroitinase ABC treatment opens a window of opportunity for task-specific rehabilitation. Nature Neuroscience 2009;12:1145. 
36. Muir EM, Fyfe I, Gardiner S, Li L, Warren P, Fawcett JW, Keynes RJ, Rogers JH. Modification of $\mathrm{N}$-glycosylation sites allows secretion of bacterial chondroitinase $\mathrm{ABC}$ from mammalian cells. Journal of Biotechnology 2010;145(2):103-110.

37. Ichiyama RM, Gerasimenko Y, Jindrich DL, Zhong H, Roy RR, Edgerton VR. Dose dependence of the 5-HT agonist quipazine in facilitating spinal stepping in the rat with epidural stimulation. Neuroscience Letters 2008;438(3):281-285.

38. Andersen T, Schmidt J, Thomassen M, Hornstrup T, Frandsen U, Randers M, Hansen P, Krustrup P, Bangsbo J. A preliminary study: Effects of football training on glucose control, body composition, and performance in men with type 2 diabetes. Scandinavian Journal of Medicine \& Science in Sports 2014.

39. Soukup T, Zachařová G, Smerdu V. Fibre type composition of soleus and extensor digitorum longus muscles in normal female inbred Lewis rats. Acta Histochemica 2002;104(4):399-405.

40. Kissane RWP, Egginton S, Askew GN. Regional variation in the mechanical properties and fibre type composition of the rat extensor digitorum longus muscle. Experimental Physiology 2017:n/a-n/a.

41. McFarlane L, Altringham JD, Askew GN. Intra-specific variation in wing morphology and its impact on take-off performance in blue tits (Cyanistes caeruleus) during escape flights. Journal of Experimental Biology 2016;219(9):1369-1377.

42. Egginton S. Anatomical adaptations for peripheral oxygen transport at high and low temperatures. South African Journal of Zoology 1998;33(2):119-128.

43. Olfert IM, Baum O, Hellsten Y, Egginton S. Advances and challenges in skeletal muscle angiogenesis. American Journal of Physiology-Heart and Circulatory Physiology 2016;310(3):H326-H336.

44. Bosutti A, Egginton S, Barnouin Y, Ganse B, Rittweger J, Degens H. Local capillary supply in muscle is not determined by local oxidative capacity. Journal of Experimental Biology 2015;218(21):3377-3380. 
Microvascular remodeling in SCI 23 
Microvascular remodeling in $\mathrm{SCl} 24$

TABLE 1. Comparison of local capillary data for individual fibre types within the TA core and cortex.

\begin{tabular}{|c|c|c|c|c|c|c|c|c|}
\hline & Core & & & & & & Cortex & \\
\hline & Type I LCFR & Type Ila LCFR & Type Ilb LCFR & $\begin{array}{c}\text { Type I LCD } \\
\left(\mu \mathrm{m}^{-2}\right)\end{array}$ & $\begin{array}{c}\text { Type Ila LCD } \\
\left(\mu \mathrm{m}^{-2}\right)\end{array}$ & $\begin{array}{c}\text { Type Ilb LCD } \\
\left(\mu \mathrm{m}^{-2}\right)\end{array}$ & Type IIb LCFR & $\begin{array}{c}\text { Type Ilb LCD } \\
\left(\mu \mathrm{m}^{-2}\right)\end{array}$ \\
\hline CT & $1.85 \pm 0.24$ & $1.54 \pm 0.04$ & $2.34 \pm 0.02$ & $1,540.2 \pm 111.7$ & $1,456.4 \pm 50.7$ & $1,244.9 \pm 54.2$ & $1.42 \pm 0.06$ & $802.9 \pm 44.2$ \\
\hline$C G$ & $1.15 \pm 0.05^{*}$ & $1.03 \pm 0.08$ * & $1.65 \pm 0.12$ * & $868.0 \pm 65.1$ * & $858.6 \pm 28.0$ * & $844.8 \pm 30.3^{*}$ & $1.24 \pm 0.08$ & $452.5 \pm 9.9$ * \\
\hline TR & $1.08 \pm 0.11^{*}$ & $1.11 \pm 0.13^{*} \dagger$ & $1.61 \pm 0.12^{*} \dagger$ & $714.0 \pm 81.8^{*}$ & $752.6 \pm 126.3^{*}$ & $690.9 \pm 96.0$ * & $1.07 \pm 0.07^{*} \dagger$ & $338.8 \pm 38.5^{*} \dagger$ \\
\hline ES & $1.54 \pm 0.09$ & $1.12 \pm 0.08^{\dagger}$ & $1.57 \pm 0.12^{*} \dagger$ & $820.0 \pm 20.5^{*}$ & $752.8 \pm 29.3^{*}$ & $749.5 \pm 46.8^{*}$ & $1.11 \pm 0.05^{*} \dagger$ & $364.4 \pm 60.1$ * \\
\hline CB & $1.42 \pm 0.10$ & $1.57 \pm 0.08^{\#}$ & $2.36 \pm 0.07^{\#}$ & $993.5 \pm 70.8^{*}$ & $1,073.8 \pm 71.1$ * & $959.6 \pm 60.4$ & $1.67 \pm 0.04^{\#}$ & $525.74 \pm 31.8^{*}$ \\
\hline
\end{tabular}


Microvascular remodeling in $\mathrm{SCl} 25$

TABLE 2. Correlation coefficients for local capillary supply data presented in Figure 4.

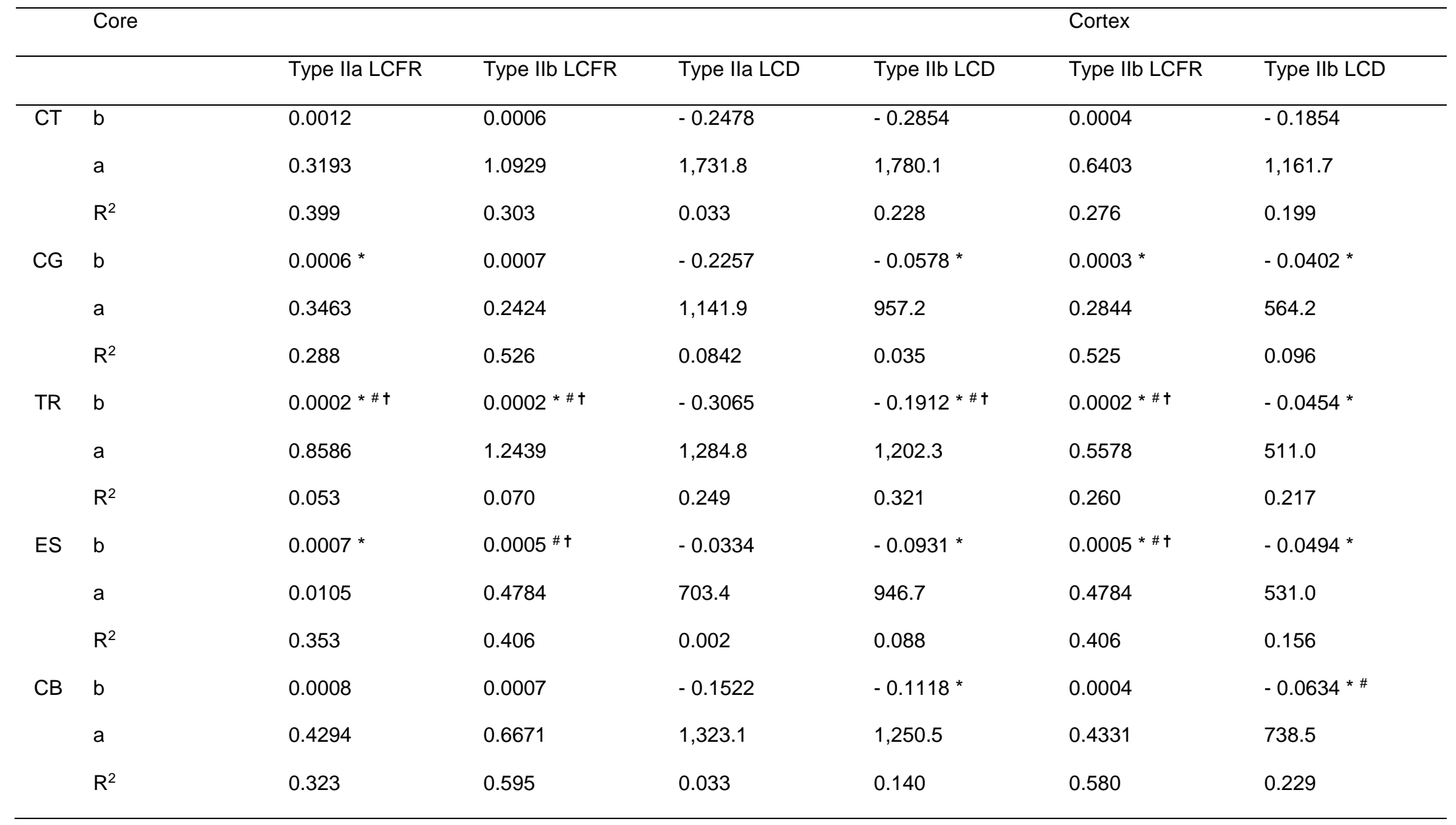


Microvascular remodeling in $\mathrm{SCl} 26$

b, slope coefficient; a, y-axis intersect; $\mathrm{R}^{2}$, Coefficient of determination. LCFR, local capillary to fibre ratio; LCD, local capillary density. CT, intact controls;

CG, spinal cord injured cage controls; TR, locomotor trained; $E S$, epidural stimulation; $C B$, combination of ES and TR. " $P<0.05$ vs. CT, \# $P<0.05$ vs. CG,

† $P<0.05$ vs. CB. Group animal numbers: CT (4), CG (4), TR (5), ES (3) and CB (4). 
A

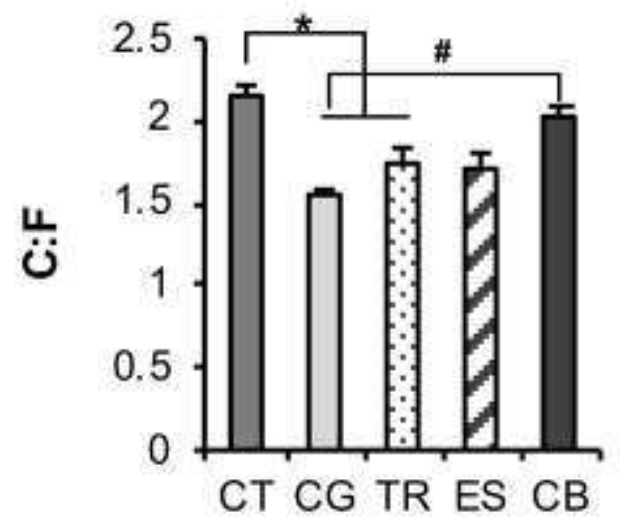

C

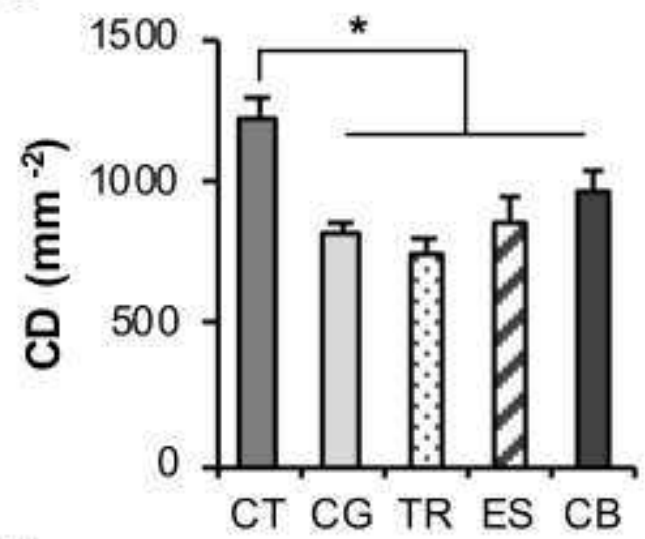

E

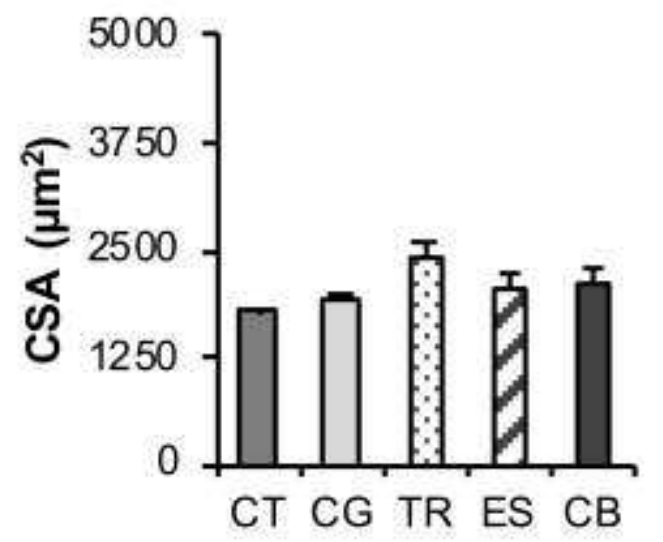

B

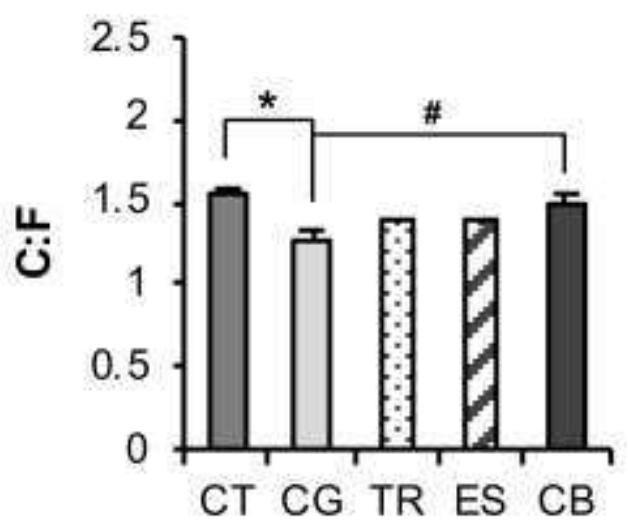

D

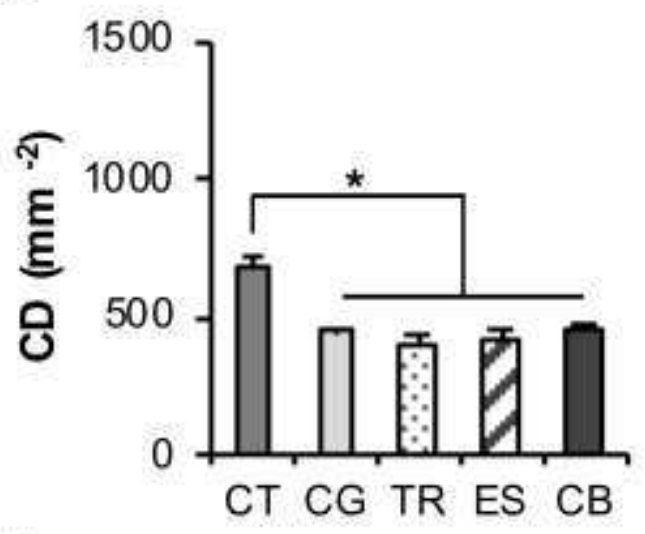

$\mathbf{F}$

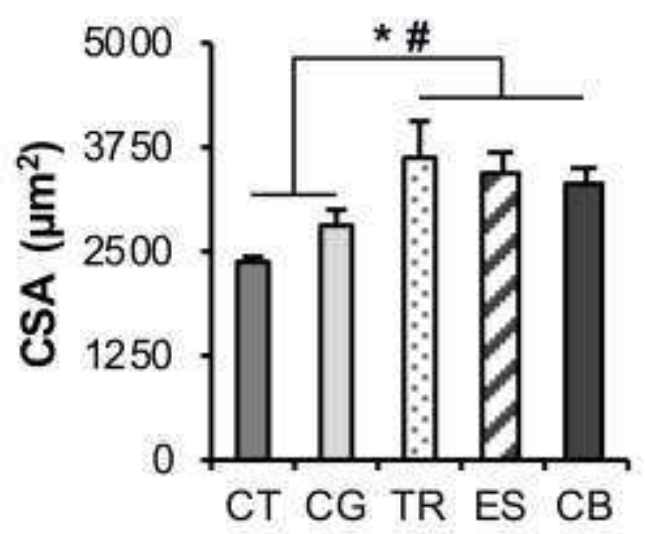

2 FIGURE 1. Global angiogenic response to $\mathrm{SCl}$ and locomotor treatment in the TA core

3 and cortex. Capillary to fibre ratio (C:F), capillary density (CD) and fibre cross sectional area 4 (CSA) are provided for the core ( $\mathrm{A}, \mathrm{C}$ and $\mathrm{E})$ and the cortex (B, D and $\mathrm{F})$, respectively. * $5 \mathrm{P}<0.05$ vs. CT, \# P<0.05 vs. CG. Group animal numbers: CT (4), CG (4), TR (5), ES (3) and $6 \mathrm{CB}(4)$. 
A

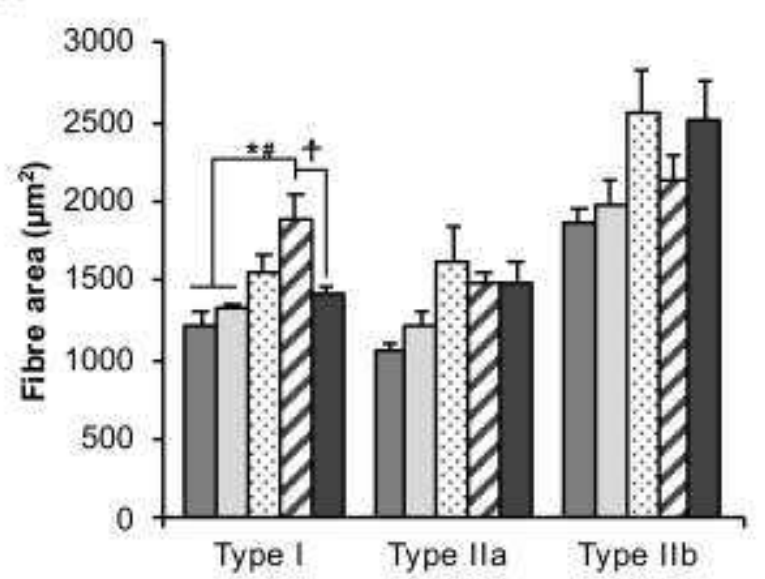

B

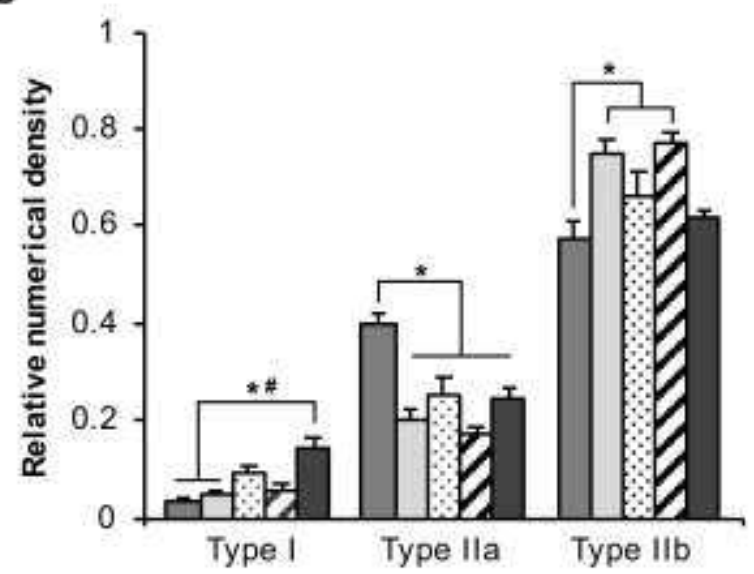

C

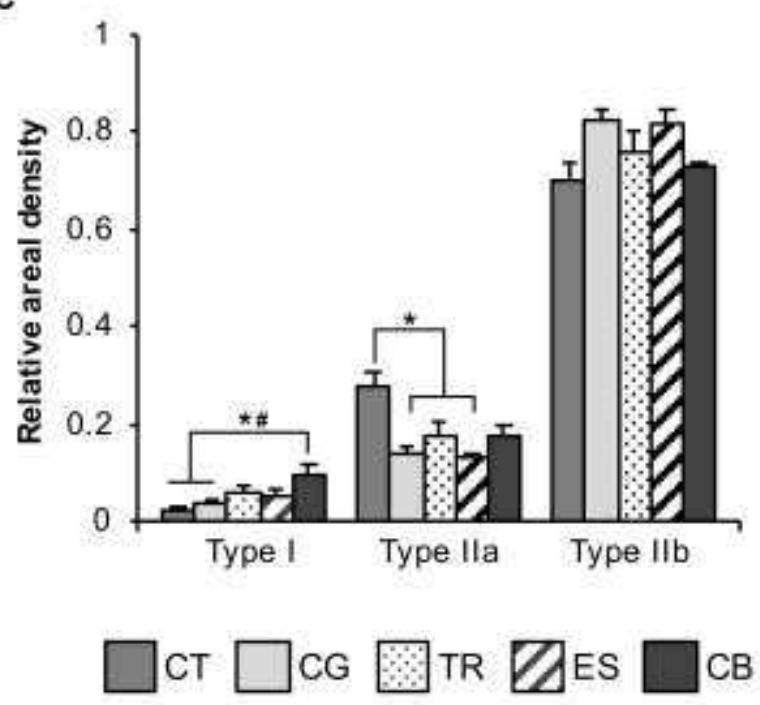

8 FIGURE 2. Fibre type composition of the TA core. Fibre type specific changes for average

9 fibre area $(A)$, relative numerical density $(B)$ and relative areal density $(C) .{ }^{*} P<0.05$ vs. $C T$, \# $10 P<0.05$ vs. CG, † P<0.05 vs. CB. Group animal numbers: CT (4), CG (4), TR (5), ES (3) and 11 CB (4). 
A

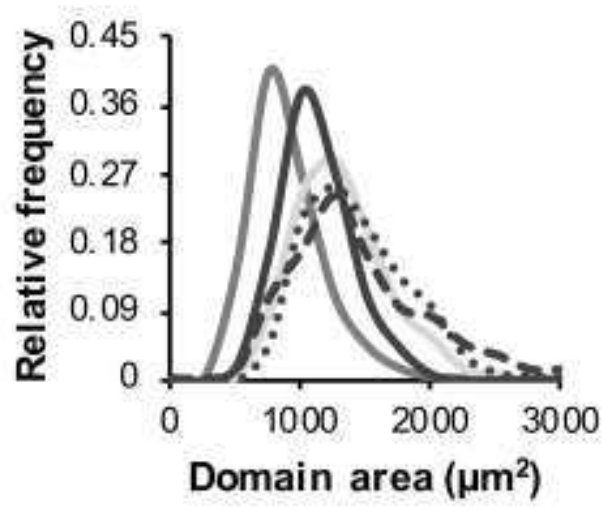

C

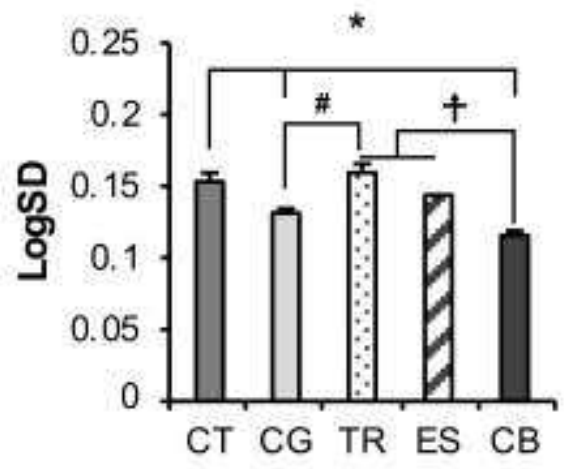

E

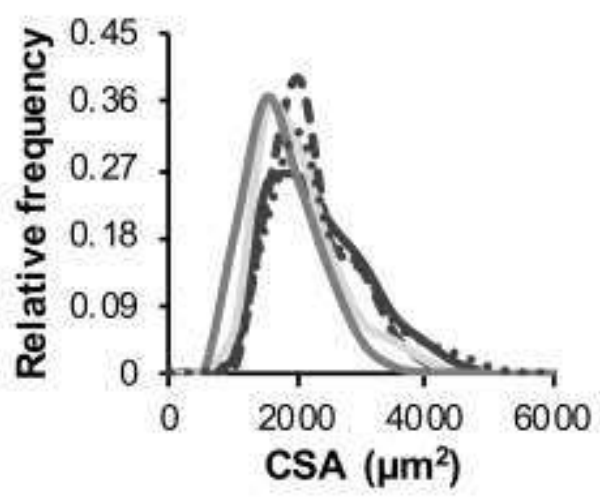

G

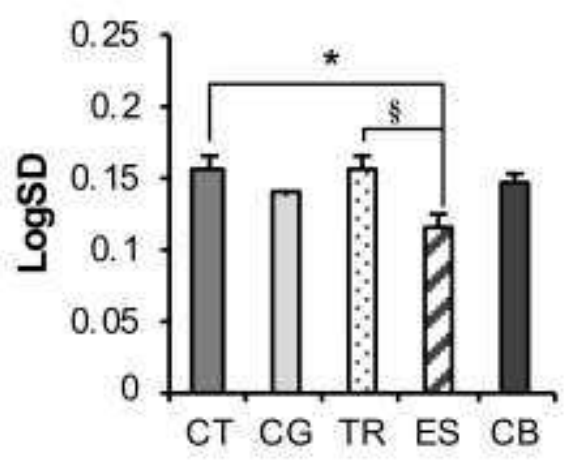

B

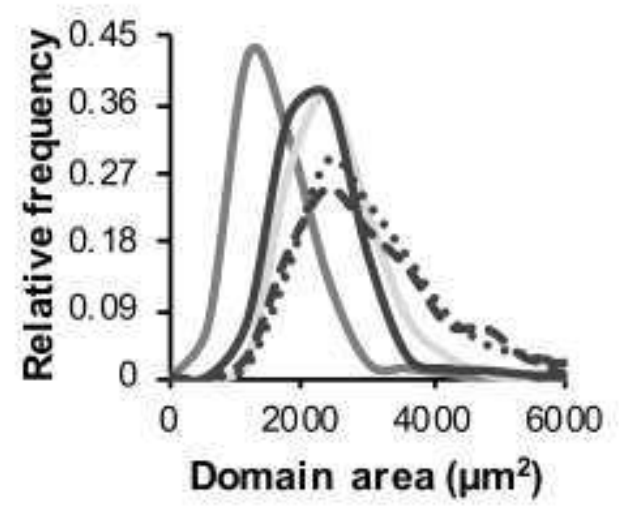

D

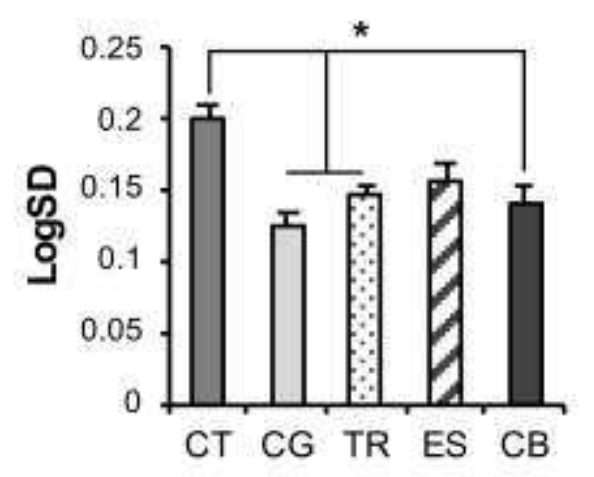

$\mathbf{F}$

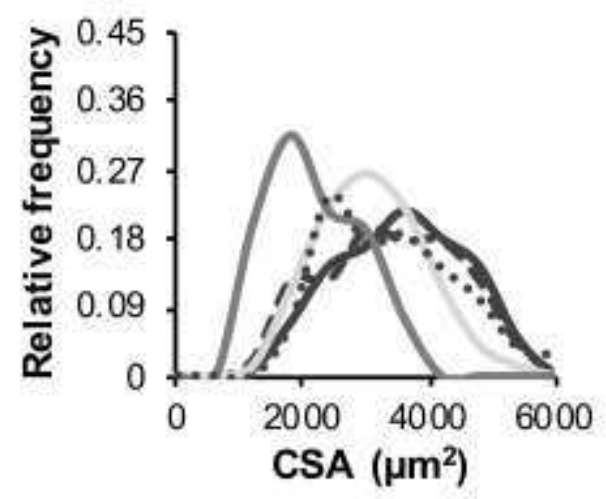

H

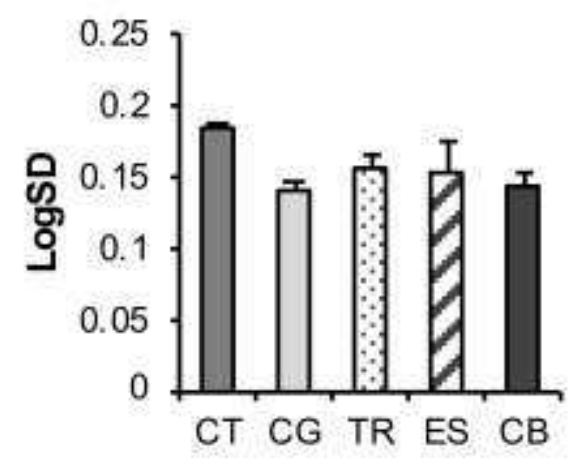


13 FIGURE 3. Capillary domain and fibre area distribution and heterogeneity for TA core

14 and cortex. Frequency distribution for capillary domain areas (A and B) and capillary 15 heterogeneity $(C$ and $D)$ presented as LogSD for the core ( $A$ and $C$ ) and cortex (B and $D)$, 16 respectively. Frequency distribution of fibre area $(E$ and $F)$ and heterogeneity of fibre size (G 17 and $\mathrm{H})$ presented as LogSD for the core $(\mathrm{E}$ and $\mathrm{G})$ and cortex $(\mathrm{F}$ and $\mathrm{H})$, respectively. ${ }^{*} \mathrm{P}<0.05$ 18 vs. $C T,{ }^{\#} \mathrm{P}<0.05$ vs. $\mathrm{CG},{ }^{\S} \mathrm{P}<0.05$ vs. $\mathrm{TR},{ }^{\dagger} \mathrm{P}<0.05$ vs. $\mathrm{CB}$. Group animal numbers: $\mathrm{CT}$ (4), 19 CG (4), TR (5), ES (3) and CB (4). 
A

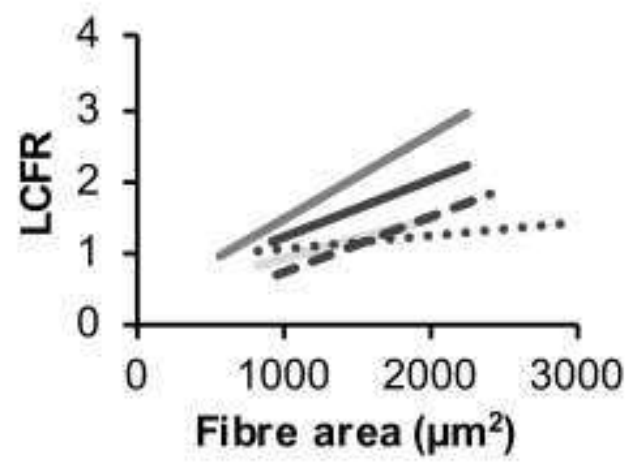

C

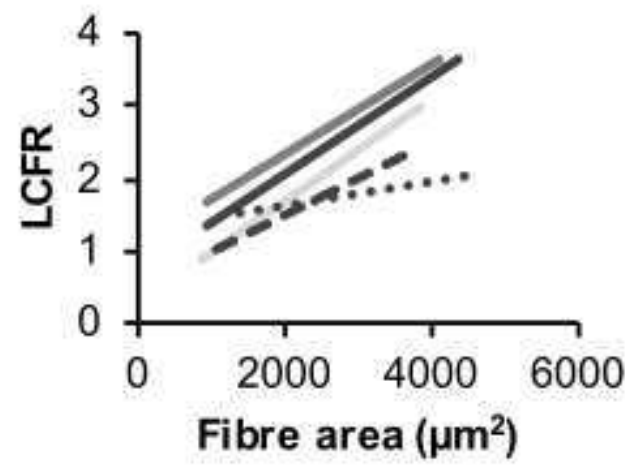

$\mathbf{E}$

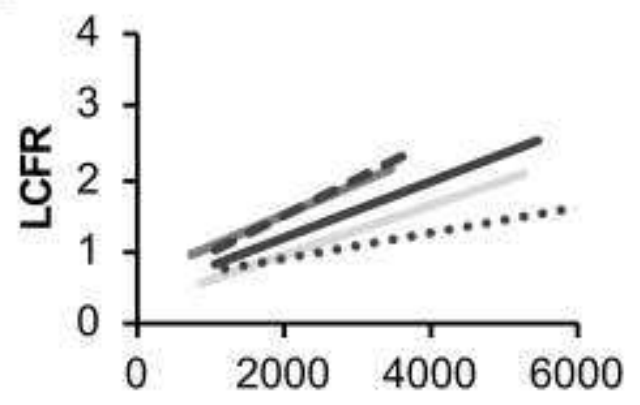

Fibre area $\left(\mu \mathrm{m}^{2}\right)$
B

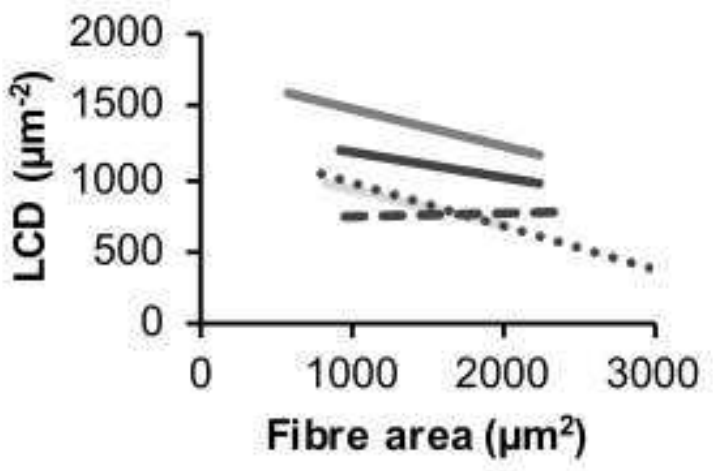

D

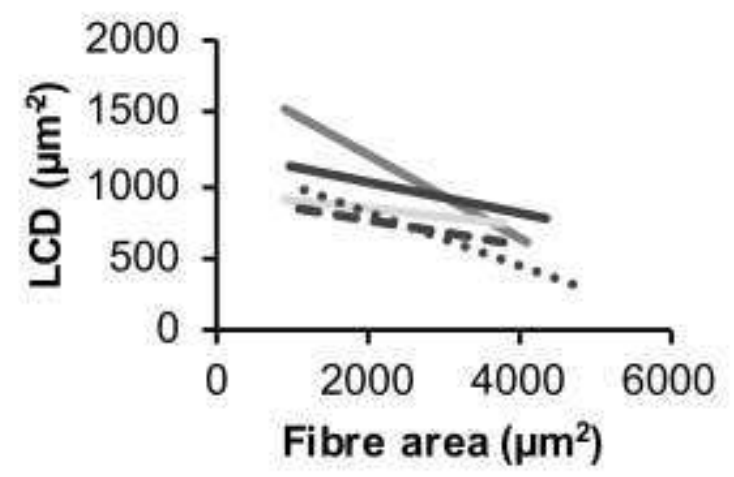

$\mathbf{F}$

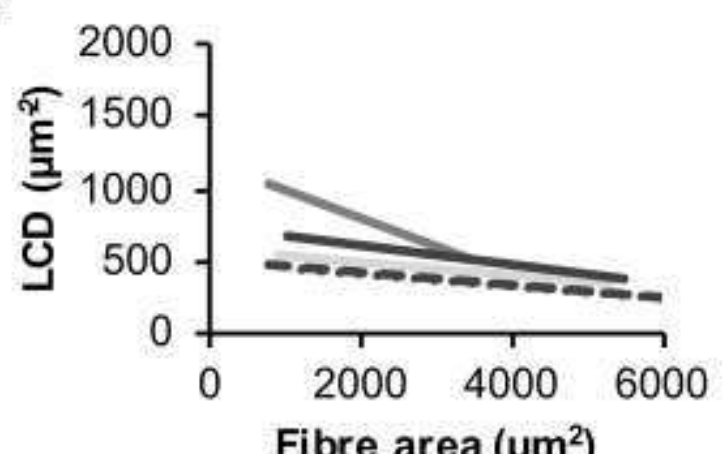

22 FIGURE 4. Local capillary supply differentiated by fibre type. LCFR (A, C and E) and $23 \operatorname{LCD}(B, D$ and $F)$ presented for Type lla fibres in the core (A and B), Type Ilb fibres in the 24 core $(\mathrm{C}$ and $\mathrm{D})$ and Type IIb in the cortex (E and F). Lines represent least-squares regression 25 fitted to all data points; note the deviation from normal allometric scaling with TR. Group animal numbers: CT (4), CG (4), TR (5), ES (3) and CB (4). 